\title{
Unraveling of gene expression control in genome-reduced bacteria. The rally goes on...
}

\author{
V. Govorun \\ Federal Research and Clinical Center of Physical-Chemical Medicine of Federal Medical Biological \\ Agency, Moscow, Russia \\ e-mail:Vgovorun@yandex.ru
}

Key words: Mycoplasma gallisepticum, gene expression regulation, RNA-seq, ribosomal profiling, cell cycle control

Mycoplasmas of class Mollicutes represent extremely reduced bacteria yet capable of self-replication without the aid of eukaryotic cell. Thus they are good models to study the basic principles of the organization of living cell. In the present work we used a combination of high-throughput technologies to elucidate the transcription control network in a model organism Mycoplasma gallisepticum. Using RNA-seq we identified transcription units and performed identification and activity quantitation of promoters of M. gallisepticum. We used comparative genomics and promoters' identification across different Mollicutes species as well as a set of perturbation models to identify putative regulatory sequences. We used ribosomal profiling and proteomics to study the transfer of genetic information to the protein level. We identified that the significant amount of transcription regulation in $M$. gallisepticum is achieved via weak determinants of the core promoter. In addition $M$. gallisepticum features conditional terminators, which may undergo read-through under the stress and contribute to transcription profile. Ribosomal profiling demonstrated that while under exponential growth translation is near equal to transcription under stress ribosome demonstrates selectivity towards particular transcripts. Analysis of cross-species promoters' conservation demonstrated that transcriptional control is largely species specific, while the most conserved transcriptional regulators control cell cycle.

Acknowledgements: This study supported by project Russian Science Foundation No. 14-24-00159. 